\title{
A Dual Quaternion Based Fusion Framework for IMU Data with 6 DOF Pose
}

\author{
Wang Yuyang, Yan Peiyi, Zhang Chunyang, Liu Zheming
}

\author{
School of Astronautics, Harbin Institute of Technology \\ 92 West Dazhi Street, Nan Gang District, Harbin, Heilongjiang Province, China \\ BCC2010@163.com
}

Keywords: Dual Quaternion; IMU; State Estimation; Kalman Filter

\begin{abstract}
Based on the highly successful application of quaternions for attitude estimation, this paper proposes an approach to position, velocity and attitude estimation for Micro Aerial Vehicles(MAVs) using dual quaternions. The states are represented in dual quaternion and time continuous states propagation model are derived via dual quaternion time update equation. At the same time, the error propagation equations based on additive error model is derived and implemented to fuse data from multiple sensors using Kalman Filter. Simulation results showed that the combination of multiple sensor data highly increase the estimate precision. In this paper, the sensor fusion algorithm is pivoted around EKF(Extended Kalman Filter) and dual quaternion.
\end{abstract}

\section{Introduction}

Micro Aerial Vehicles(MAVs) will play an increasingly important role in disaster management, industrial inspection and environment conservation. For such operations, navigating system onboard provides estimates of their states and even environment information to perform tasks accurately and agilely. For minimal cost and weight, Inertial Measurement Unit (IMU) is generally implemented in combination with other sensors.

This paper proposed a fusion framework using IMU with other sensors based on dual quaternion for MAVs. Dual quaternion is a powerful mathematical tool representing translation and rotation transformations of rigid body and have been shown to be the most efficient and most compact form of representing rigid body motion[5]. The inertial motion model have been exploited in detail in references [1][3] and the model is unified in dual quaternion formulation. The proposed expressions are verified through experiment with simulated data. Further, dual quaternion error state differential equations are derived used in EKF framework to fuse data from multiple sources.

Subsequent sections are structured as follows. Section Mathematical Preliminaries briefly explains the theory on quaternion, dual number, dual quaternion and its representation of rigid body motion in 3D space. Section State Estimation for MAVs describes inertial motion model in the formulation of dual quaternion and the fusion framework based on EKF. Section Simulation and Discussion presents simulation results and discussions. Finally section Conclusion concludes the work.

\section{Mathematical Preliminaries}

This chapter presents the introduction to dual quaternion. 


\subsection{Quaternion}

The quaternion is generally defined as

$$
q=q_{0}+q_{1} \mathbf{i}+q_{2} \mathbf{j}+q_{3} \mathbf{k} .
$$

The quantity $q_{0}$ is the real or scalar part of the quaternion, and $q_{1} \mathbf{i}+q_{2} \mathbf{j}+q_{3} \mathbf{k}$ is the imaginary or vector part. The quaternion can therefore also be written in a four-dimensional column matrix

$$
q=\left[\begin{array}{llll}
q_{0} & q_{1} & q_{2} & q_{3}
\end{array}\right]^{T} .
$$

Quaternion can also be represented as $q=[s, \mathbf{v}]$ and $q=\left[\cos \frac{\theta}{2}, \sin \frac{\theta}{2} \mathbf{v}\right]$ where $\theta$ is the angle of rotation and $\mathbf{v}$ is the axis of rotation. Unit norm quaternion rotates a vector about quaternion's vector. It is given by $q \mathbf{p} q^{-1}$ where $\mathbf{p}$ is the vector to be rotated and $q$ is the unit norm quaternion. For unit norm quaternion, $q^{-1}=q^{*}$. Multiplication of quaternions results another quaternion which is given by

$$
\begin{aligned}
q_{a} \otimes q_{b} & =\left[s_{a}, \mathbf{a}\right]\left[s_{b}, \mathbf{b}\right] \\
& =\left[s_{a} s_{b}-\mathbf{a} \cdot \mathbf{b}, s_{a} \mathbf{b}+s_{b} \mathbf{a}+\mathbf{a} \times \mathbf{b}\right] .
\end{aligned}
$$

where $\otimes$ is the quaternion multiplication, $\mathbf{a} \cdot \mathbf{b}$ is the dot product and $\mathbf{a} \times \mathbf{b}$ is cross product of vectors $\mathbf{a}$ and $\mathbf{b}$. It is to be noted that quaternion multiplication is not commutative. Quaternion multiplication can also be represented as a 4-element vector multiplied by a matrix.

$$
\begin{aligned}
q_{c} & =q_{a} \otimes q_{b}=R\left(q_{b}\right) q_{a} \\
& =\left[\begin{array}{cccc}
q_{b 0} & -q_{b 1} & -q_{b 2} & -q_{b 3} \\
q_{b 1} & q_{b 0} & q_{b 3} & -q_{b 2} \\
q_{b 2} & -q_{b 3} & q_{b 0} & q_{b 1} \\
q_{b 3} & q_{b 2} & -q_{b 1} & q_{b 0}
\end{array}\right]\left[\begin{array}{l}
q_{a 0} \\
q_{a 1} \\
q_{a 2} \\
q_{a 3}
\end{array}\right] . \\
q_{c} & =q_{a} \otimes q_{b}=L\left(q_{a}\right) q_{b} \\
& =\left[\begin{array}{cccc}
q_{a 0} & -q_{a 1} & -q_{a 2} & -q_{a 3} \\
q_{a 1} & q_{a 0} & -q_{a 3} & q_{a 2} \\
q_{a 2} & q_{a 3} & q_{a 0} & -q_{a 1} \\
q_{a 3} & -q_{a 2} & q_{a 1} & q_{a 0}
\end{array}\right]\left[\begin{array}{l}
q_{b 0} \\
q_{b 1} \\
q_{b 2} \\
q_{b 3}
\end{array}\right] .
\end{aligned}
$$

The Jacobian matrix of $q_{c}$ with respect to $q_{a}$ and $q_{b}$ respectively are:

$$
\begin{aligned}
& \frac{\partial q_{c}}{\partial q_{a}}=R\left(q_{b}\right) . \\
& \frac{\partial q_{c}}{\partial q_{b}}=L\left(q_{a}\right) .
\end{aligned}
$$

The properties of quaternion algebra are discussed more in detail in [6]. 


\subsection{Dual Number}

Dual number is similar to a complex number and it is defined as,

$$
\widehat{Z}=Z+\varepsilon Z^{\circ} .
$$

where $z$ is the real part, $z^{o}$ is the dual part and $\varepsilon$ is the dual operator. These real and dual part can be a real number, vector or matrix. The dual number framework is used in kinematics to represent rigid body displacement in 3D space.

Dual number supports all algebraic properties and operations. The product of two dual number results another dual number which is denoted as,

$$
\widehat{z}_{a} \widehat{z}_{b}=z_{a} z_{b}+\varepsilon\left(z_{a} b^{0}+a^{0} z_{b}\right) .
$$

Dual matrix can be defined as $\widehat{\mathbf{A}}=\mathbf{A}+\varepsilon \mathbf{A}^{o}$ where each element of the matrix is dual number. The product of two dual matrices is given by,

$$
\widehat{\mathbf{A}} \hat{\mathbf{B}}=\mathbf{A B}+\varepsilon\left(\mathbf{A B}^{o}+\mathbf{A}^{o} \mathbf{B}^{o}\right) .
$$

\subsection{Dual Quaternion}

Dual quaternion $\hat{q}$ is a dual number with quaternion components. It is defined as,

$$
\widehat{q}=q_{r}+\varepsilon q_{d}^{o}
$$

where $q_{r}$ and $q_{d}^{o}$ are quaternions and real and dual component of dual quaternion respectively. Each dual quaternion consists of eight scalar elements. Pair of quaternions are represented in single dual quaternion variable. It provides a compact way of representation and rapid algebraic operations. The addition of two dual quaternions can be represented as

$$
\widehat{q}_{1}+\widehat{q}_{2}=\left(q_{r 1}+q_{r 2}\right)+\varepsilon\left(q_{d 1}^{o}+q_{d 2}^{o}\right) .
$$

and the multiplication is defined as,

$$
\widehat{q}_{1} \widehat{q}_{2}=\left(q_{r 1} \otimes q_{r 2}\right)+\varepsilon\left(q_{r 1} \otimes q_{d 2}^{o}+q_{d 1}^{o} \otimes q_{r 2}\right) .
$$

Dual quaternion supports all normal algebraic properties and operations [9]. The conjugate of a dual quaternion is $\hat{q}^{*}=q_{r}^{*}+\varepsilon q_{d}^{0^{*}}$. The product of $\hat{q}$ and its conjugate $\hat{q}^{*}$ is one for unit dual quaternion, $\widehat{q}^{*}{ }^{*}=\widehat{q}^{*} \hat{q}=1$. Unit dual quaternion is extensively used in kinematics to represent transformation. The inverse of a dual quaternion is given by

$$
\hat{q}^{-1}=q_{r}^{-1}-\varepsilon\left(q_{r}^{-1} q_{d}^{o} q_{r}^{-1}\right) .
$$

The motion of rigid body in $3 \mathrm{D}$ space involves rotation followed by translation at every time instant. The rigid body transformation of a body frame with another frame, e.g. inertial frame can be represented compactly by a unit dual quaternion as follows, 


$$
\begin{aligned}
\widehat{q}_{i b} & =q_{i b, r}+\varepsilon q_{i b, t}^{o} \\
& =q_{i b, r}+\varepsilon \frac{1}{2} t_{i b}^{i} \otimes q_{i b, r} \\
& =q_{i b, r}+\varepsilon \frac{1}{2} q_{i b, r} \otimes t_{i b}^{b} .
\end{aligned}
$$

The subscript identifies the rotation or translation of body frame $b$ with respect to base frame $i . q_{i b, r}$ is the unit quaternion represents rotation and $t_{i b}^{i}=\left[\begin{array}{ll}0 & \mathbf{t}_{i b}^{i T}\end{array}\right]^{T}, t_{i b}^{b}=\left[\begin{array}{ll}0 & \mathbf{t}_{i b}^{b T}\end{array}\right]^{T}, \quad \mathbf{t}_{i b}^{i} \quad$ is translation vector in frame $i$ and $\mathbf{t}_{i b}^{b}$ in frame $b$.

The rotational and translational kinematic equations written in terms of dual quaternions can be written compactly as[4]

$$
\dot{\hat{q}}=\frac{1}{2} \widehat{q}_{i b} \otimes \widehat{\omega}_{i b}^{b}=\frac{1}{2} \widehat{\omega}_{i b}^{i} \otimes \widehat{q}_{i b}
$$

with $\hat{\omega}_{i b}^{b}$ the dual velocity of frame $b$ with respect to frame $i$ expressed in frame $b$ and $\widehat{\omega}_{i b}^{i}$ in frame $i . \hat{\omega}_{i b}^{b}$ and $\hat{\omega}_{i b}^{i}$ are defined as

$$
\begin{gathered}
\widehat{\omega}_{i b}^{b}=\omega_{i b}^{b}+\varepsilon v_{i b}^{b} . \\
\widehat{\omega}_{i b}^{i}=\omega_{i b}^{i}+\varepsilon\left(v_{i b}^{i}-\omega_{i b}^{i} \times t_{i b}^{i}\right) .
\end{gathered}
$$

with $\omega_{i b}^{b}=\left[\begin{array}{ll}0 & \boldsymbol{\omega}_{i b}^{b T}\end{array}\right]^{T}, \quad v_{i b}^{b}=\left[\begin{array}{ll}0 & \mathbf{v}_{i b}^{b T}\end{array}\right]^{T}, \quad \omega_{i b}^{i}=\left[\begin{array}{ll}0 & \boldsymbol{\omega}_{i b}^{i T}\end{array}\right]^{T}, \quad v_{i b}^{i}=\left[\begin{array}{ll}0 & \mathbf{v}_{i b}^{i T}\end{array}\right]^{T}, \boldsymbol{\omega}_{i b}^{b} \quad$ and $\quad \boldsymbol{\omega}_{i b}^{i} \quad$ are angular velocity of frame $b$ with respect to frame $i$ expressed in frame $b$ and frame $i$ respectively . $\mathbf{v}_{i b}^{b}$ and $\mathbf{v}_{i b}^{i}$ are linear velocity of frame $b$ with respect to frame expressed in frame $b$. and frame $i$ respectively.

\section{State Estimation for MAVs}

An EKF framework generally consists of prediction and update step. The prediction step is responsible for propagating the state from one time step to next and new measurement information from update step can correct the predicted state with more precision.

\subsection{Inertial sensor model}

Inertial Measurement Unit(IMU) comprises 3-axis accelerometer and 3-axis gyro providing accelerations and angular velocities in body frame. The inertial measurements are assumed to contain a bias $\mathbf{b}$ and a zero mean Gaussian white noise $\mathbf{n}$. Thus, real accelerations and angular velocities can be obtained by

$$
\begin{aligned}
& \boldsymbol{\omega}=\boldsymbol{\omega}_{m}-\mathbf{b}_{\omega}-\mathbf{n}_{\omega} . \\
& \mathbf{a}=\mathbf{a}_{m}-\mathbf{b}_{a}-\mathbf{n}_{a} .
\end{aligned}
$$

with $\omega_{m}$ as gyro measurements, $\boldsymbol{a}_{m}$ as accelerometer measurements, $\mathbf{b}_{\omega}$ as gyro bias and $\mathbf{b}_{a}$ as accelerometer. The dynamics of a non-static bias $\mathbf{b}$ are modeled as a random process:

$$
i \dot{\mathbf{b}}_{\omega}=\mathbf{n}_{b_{\omega}} .
$$




$$
\dot{\mathbf{b}}_{a}=\mathbf{n}_{b_{a}} .
$$

with $\mathbf{n}_{b_{\omega}}$ and $\mathbf{n}_{b_{a}}$ as zero mean Gaussian white noise.

We assume the bias $\mathbf{b}$ is static.

$$
\begin{aligned}
& \dot{\mathbf{b}}_{\omega}=0 . \\
& \dot{\mathbf{b}}_{a}=0 .
\end{aligned}
$$

The accelerations and angular velocities can be expressed in terms of quaternion:

$$
\begin{aligned}
& q_{\boldsymbol{\omega}}=q_{\boldsymbol{\omega}_{m}}-q_{\mathbf{b}_{\omega}}-q_{\mathbf{n}_{\omega}} . \\
& q_{\mathbf{a}}=q_{\mathbf{a}_{m}}-q_{\mathbf{b}_{a}}-q_{\mathbf{n}_{a}} .
\end{aligned}
$$

where $q_{\mathbf{v}}=\left[\begin{array}{ll}0 & \mathbf{v}^{T}\end{array}\right]^{T}$ and $\mathbf{v}$ is a $3 \times 1$ vector.

\subsection{Inertial Motion Model}

[1][2] proposed an IMU-vision data fusion framework based on EKF in detail. We simplify the state definition in the reference. The states are stacked into a 20-element vector and consist of position $q_{i b, p}$, velocity $q_{i b, v}$ in inertial frame and rotation $q_{i b, r}$ describing rotation of IMU body frame with respect to inertial frame in terms of quaternion. The state vector can be written as a vector:

$$
\mathbf{X}=\left[q_{i b, p}^{T}, q_{i b, v}^{T}, q_{i b, r}^{T}, \mathbf{b}_{\boldsymbol{\omega}}^{T}, \mathbf{b}_{\mathbf{a}}^{T}\right]^{T} .
$$

The time continuous differential equations can be obtained through (16):

$$
\begin{gathered}
\dot{q}_{i b, p}=\frac{1}{2} q_{i b, p} \otimes\left(q_{\boldsymbol{\omega}_{m}}-q_{\mathbf{b}_{\omega}}-q_{\mathbf{n}_{\omega}}\right)+q_{i b, v} . \\
\dot{q}_{i b, v}=\frac{1}{2} q_{i b, v} \otimes\left(q_{\boldsymbol{\omega}_{m}}-q_{\mathbf{b}_{\omega}}-q_{\mathbf{n}_{\omega}}\right)+ \\
\frac{1}{2} q_{i b, r} \otimes\left(q_{\mathbf{a}_{m}}-q_{\mathbf{b}_{a}}-q_{\mathbf{n}_{a}}\right)+\frac{1}{2} q_{\mathbf{g}} \otimes q_{i b, r} . \\
\dot{q}_{i b, r}=\frac{1}{2} q_{i b, r} \otimes\left(q_{\boldsymbol{\omega}_{m}}-q_{\mathbf{b}_{\omega}}-q_{\mathbf{n}_{\omega}}\right) . \\
\dot{\mathbf{b}}_{\omega}=\mathbf{n}_{b_{\omega}} . \\
\dot{\mathbf{b}}_{a}=\mathbf{n}_{b_{a}} .
\end{gathered}
$$

where $q_{\mathrm{g}}=\left[\begin{array}{llll}0 & 0 & 0 & -g_{0}\end{array}\right]^{T}$ and $g_{0}$ is local gravity.

Taking the expectations of the above derivatives, we obtain

$$
\hat{\dot{q}}_{i b, p}=\frac{1}{2} \hat{q}_{i b, p} \otimes\left(q_{\omega_{m}}-\hat{q}_{\mathbf{b}_{\omega}}\right)+\hat{q}_{i b, v} .
$$




$$
\begin{gathered}
\hat{\dot{q}}_{i b, v}=\frac{1}{2} \hat{q}_{i b, v} \otimes\left(q_{\boldsymbol{\omega}_{m}}-\hat{q}_{\mathbf{b}_{\omega}}\right)+\frac{1}{2} \hat{q}_{i b, r} \otimes\left(q_{\mathbf{a}_{m}}-\hat{q}_{\mathbf{b}_{a}}\right) \\
+\frac{1}{2} q_{\mathbf{g}} \otimes \hat{q}_{i b, r .} \\
\hat{\dot{q}}_{i b . r}=\frac{1}{2} \hat{q}_{i b, r} \otimes\left(q_{\boldsymbol{\omega}_{m}}-\hat{q}_{\mathbf{b}_{\omega}}\right) . \\
\hat{\dot{\mathbf{b}}}_{\omega}=\mathbf{n}_{b_{\omega}} . \\
\hat{\dot{\mathbf{b}}}_{a}=\mathbf{n}_{b_{a}} .
\end{gathered}
$$

\subsection{Error State Model}

In the above state representation, the position, velocity and attitude of IMU frame are represented in terms of dual quaternion. It is common that the error states are implemented in the EKF framework. Therefore, we define the additive error state representation as $\delta q=q-\hat{q}$ with $q$ as the real quaternion and $\hat{q}$ as the estimate quaternion. The error state can be expressed in a vector:

$$
\delta \mathbf{X}=\left[\delta q_{i b, p}^{T}, \delta q_{i b, v}^{T}, \delta q_{i b, r}^{T}, \delta \mathbf{b}_{\omega}^{T}, \delta \mathbf{b}_{\mathbf{a}}^{T}\right]^{T}
$$

as the difference of estimate to its quantity, e.g. $\delta q_{i b, p}=q_{i b, p}-\hat{q}_{i b, p}$. The differential equations for the continuous time error state are

$$
\begin{aligned}
\delta \dot{q}_{i b, p}= & \frac{1}{2} \delta q_{i b, p} \otimes\left(q_{\boldsymbol{\omega}_{m}}-\hat{q}_{\mathbf{b}_{\omega}}\right)+\delta q_{i b, v} \\
& -\frac{1}{2} \hat{q}_{i b, p} \otimes \delta q_{\mathbf{b}_{\omega}}-\frac{1}{2} \hat{q}_{i b, p} \otimes \delta q_{\mathbf{n}_{\omega}} . \\
\delta \dot{q}_{i b, v}= & \frac{1}{2} \delta q_{i b, v} \otimes\left(q_{\boldsymbol{\omega}_{m}}-\hat{q}_{\mathbf{b}_{\omega}}\right)-\frac{1}{2} \hat{q}_{i b, v} \otimes q_{\mathbf{n}_{\omega}} \\
& +\frac{1}{2} q_{\mathbf{g}} \otimes \delta q_{i b, r}-\frac{1}{2} \hat{q}_{i b, v} \otimes \delta q_{\mathbf{b}_{\omega}} \\
& +\frac{1}{2} \delta q_{i b, r} \otimes\left(q_{\mathbf{a}_{m}}-\hat{q}_{\mathbf{b}_{a}}\right)-\frac{1}{2} \hat{q}_{i b, r} \otimes q_{\mathbf{n}_{a}} \\
& -\frac{1}{2} \hat{q}_{i b, r} \otimes \delta q_{\mathbf{b}_{a}} \cdot \\
\delta \dot{q}_{i b, r}= & \frac{1}{2} \delta q_{i b, r} \otimes\left(q_{\boldsymbol{\omega}_{m}}-\hat{q}_{\mathbf{b}_{\omega}}\right)-\frac{1}{2} \hat{q}_{i b, r} \otimes \delta q_{\mathbf{b}_{\omega}} \\
& -\frac{1}{2} \hat{q}_{i b, r} \otimes q_{\mathbf{n}_{\omega}} \cdot \\
\delta \dot{\mathbf{b}}_{\omega}= & \mathbf{n}_{b_{\omega}} \cdot \\
\delta \dot{\mathbf{b}}_{a}= & \mathbf{n}_{b_{a}} \cdot
\end{aligned}
$$


These equations can be linearized via Taylor expansion and can be written compactly:

$$
\delta \dot{\mathbf{X}}=\mathbf{F}_{c} \delta \mathbf{X}+\mathbf{G}_{c} \mathbf{n}
$$

with $\mathbf{n}$ being the noise vector $\mathbf{n}=\left[\mathbf{n}_{a}^{T}, \mathbf{n}_{b_{a}}^{T}, \mathbf{n}_{\omega}^{T}, \mathbf{n}_{b_{\omega}}^{T}\right]^{T}$.

$\mathbf{F}_{c}$ is

$\mathbf{F}_{c}=\left[\begin{array}{ccccc}\frac{1}{2} R\left(q_{\boldsymbol{\omega}_{m}}-\hat{q}_{\mathbf{b}_{\omega}}\right) & I_{4} & 0_{4} & -\frac{1}{2} L\left(\hat{q}_{i b, p}\right)_{i, 2: 4} & 0_{4 \times 3} \\ 0_{4} & \frac{1}{2} R\left(q_{\boldsymbol{\omega}_{m}}-\hat{q}_{\mathbf{b}_{\omega}}\right) & \frac{1}{2}\left[R\left(q_{\mathbf{a}_{m}}-\hat{q}_{\mathbf{b}_{a}}\right)+L\left(q_{\mathbf{g}}\right)\right]-\frac{1}{2} L\left(\hat{q}_{i b, v}\right)_{:, 2: 4} & -\frac{1}{2} L\left(\hat{q}_{i b, r}\right)_{: 22: 4} \\ 0_{4} & 0_{4} & \frac{1}{2} R\left(q_{\boldsymbol{\omega}_{m}}-\hat{q}_{\mathbf{b}_{\omega}}\right) & -\frac{1}{2} L\left(\hat{q}_{i r}^{b}\right)_{: 2: 4} & 0_{4 \times 3} \\ 0_{6 \times 4} & 0_{6 \times 4} & 0_{6 \times 4} & 0_{6 \times 3} & 0_{6 \times 3}\end{array}\right]$.

$\mathbf{F}_{c}$ can be discretized by:

$$
\begin{aligned}
\mathbf{F}_{d} & =\exp \left(\mathbf{F}_{c} \Delta t\right) \\
& =I_{d}+\mathbf{F}_{c} \Delta t+\frac{1}{2 !} \mathbf{F}_{c}^{2} \Delta t^{2}+\cdots .
\end{aligned}
$$

is

$$
\boldsymbol{G}_{c}=\left[\begin{array}{cccc}
0_{4 \times 3} & 0_{4 \times 3} & -\frac{1}{2} L\left(\hat{q}_{i b, p}\right)_{; 2,24} & 0_{6 \times 3} \\
-\frac{1}{2} L\left(\hat{q}_{i b, r}\right)_{: 224} & 0_{4 \times 3} & -\frac{1}{2} L\left(\hat{q}_{i b, v}\right)_{: 2,24} & 0_{6 \times 3} \\
0_{4 \times 3} & 0_{4 \times 3} & -\frac{1}{2} L\left(\hat{q}_{i b, r}\right)_{i 2,24} & 0_{6 \times 3} \\
0_{3} & 0_{3} & 0_{3} & I_{3} \\
0_{3} & I_{3} & 0_{3} & 0_{3}
\end{array}\right] .
$$

with $R(\bullet)$ from $(6), L(\bullet)$ from (7) and the subscript :,2:4 identifying that the column vectors spanning from index 2 to 4 are specified as a new matrix.

The discrete-time covariance matrix $\boldsymbol{Q}_{d}$ can be obtained

$$
\boldsymbol{Q}_{d}=\int_{\Delta t} \boldsymbol{F}(\tau) \boldsymbol{G}_{c} \boldsymbol{Q}_{c} \boldsymbol{G}_{c}^{T} \boldsymbol{F}(\tau)^{T} d \tau
$$

with the $\boldsymbol{Q}_{c}$ being the continuous- $\mathbf{G}_{c}$ time covariance matrix $\mathbf{Q}_{c}=\operatorname{diag}\left(\boldsymbol{\sigma}_{n_{a}}^{2}, \boldsymbol{\sigma}_{n_{b_{a}}}^{2}, \boldsymbol{\sigma}_{n_{\omega}}^{2}, \boldsymbol{\sigma}_{n_{b_{\omega}}}^{2}\right)$. Therefore, the prior covariance matrix $\mathbf{P}_{k / k-1}$ associated with the prediction of the state is provided:

$$
\mathbf{P}_{k / k-1}=\mathbf{F}_{d} \mathbf{P}_{k-1} \mathbf{F}_{d}^{T}+\mathbf{Q}_{d} .
$$

where $\mathbf{P}_{k-1}$ denotes the posterior estimate of error covariance matrix at time $k-1$.

\subsection{Measurement Model}

A 6-DOF (Degree Of Freedom) pose can be obtained from camera or radar and used here to 
measurement system state. We define measurement $\mathbf{Z}$ as the difference between actual measurements and the predicted values:

$$
\mathbf{Z}=\left[\begin{array}{c}
q_{i b, p}^{m} \\
q_{i b, r}^{m}
\end{array}\right]-\left[\begin{array}{l}
\hat{q}_{i b, p} \\
\hat{q}_{i b, r}
\end{array}\right] .
$$

with superscript $m$ identifying measurement data. It is assumed that the measurement position and rotation quaternion is aligned in inertial frame.

The measurement model that maps the state vector into measurement can be derived by linearization as:

$$
\mathbf{Z}_{k}=\mathbf{H} \delta \mathbf{X}_{k / k-1}+\mathbf{V}_{k}
$$

with $\mathbf{V}_{k}$ being zero mean Gaussian white noise at time $k$, the subscript $k / k-1$ identifying the predicted state at time $k$ and $\mathbf{H}$ as:

$$
\mathbf{H}=\left[\begin{array}{cccc}
I_{4} & 0_{4} & 0_{4} & 0_{4 \times 6} \\
0_{4} & 0_{4} & I_{4} & 0_{4 \times 6}
\end{array}\right] .
$$

The noise with the measurement model is zero Gaussian white noise and covariance matrix $\mathbf{R}=\operatorname{diag}\left(\boldsymbol{\sigma}_{q_{i b, p}^{m}}^{2}, \boldsymbol{\sigma}_{q_{i b, r}^{m}}^{2}\right)$ representing the measurement uncertainty.

The Kalman gain is given by,

$$
\mathbf{K}_{k}=\mathbf{P}_{k / k-1} \mathbf{H}^{T}\left(\mathbf{H P}_{k / k-1} \mathbf{H}^{T}+\mathbf{R}\right)^{-1} .
$$

The posterior estimate of error covariance matrix is

$$
\mathbf{P}_{k}=\left(\mathbf{I}-\mathbf{K}_{k} \mathbf{H}\right) \mathbf{P}_{k / k-1} .
$$

\section{Simulation And Discussions}

A dual quaternion based fusion framework has been proposed and experimented with simulation data. We generate 3-axis sinusoidal trajectory and attitude angle data using equations in reference [1].

\subsection{Simulation Without Noise}

In the experiment, a dual quaternion based state propagation method has been compared with quaternion based approach. The time-continuous differential equations (28) (32) are discretized and integrated using the generated gyro and accelerometer data without adding any noise. Fig. 1 and Fig. 2 Velocity error using IMU simulated data without adding noise show the performance of dual quaternion based inertial dynamics method over quaternion based method. The position and velocity error in altitude direction through single step integral of set of quaternion based dynamics equations can have a divergence rate far beyond the proposed method and in other two directions the performance of two methods is similar although the proposed method is inferior to the quaternion based approach. It can be concluded that dual quaternion based method captures the coupling between rotation and translation more accurately. 

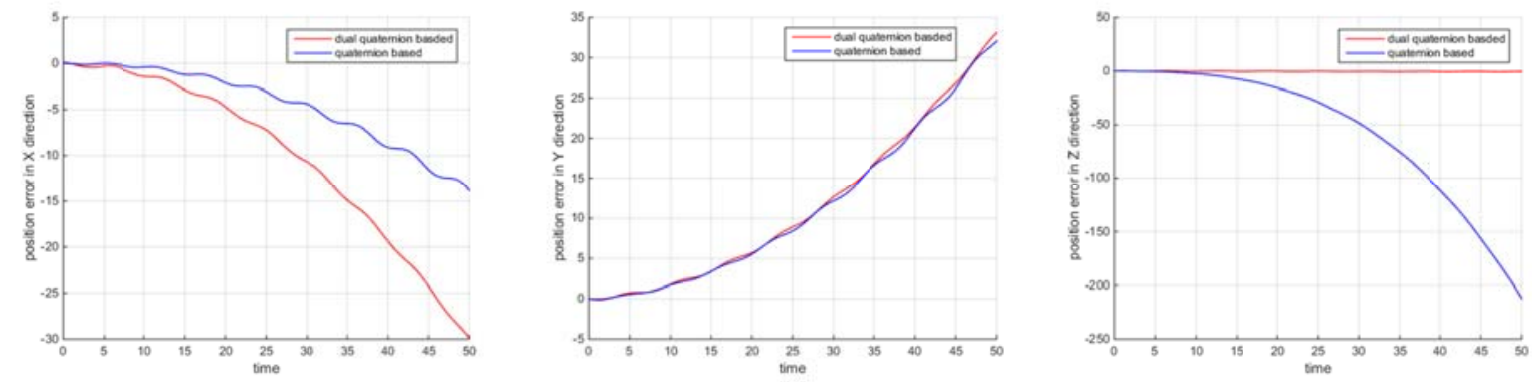

Fig. 1 Position error using IMU simulated data without adding noise
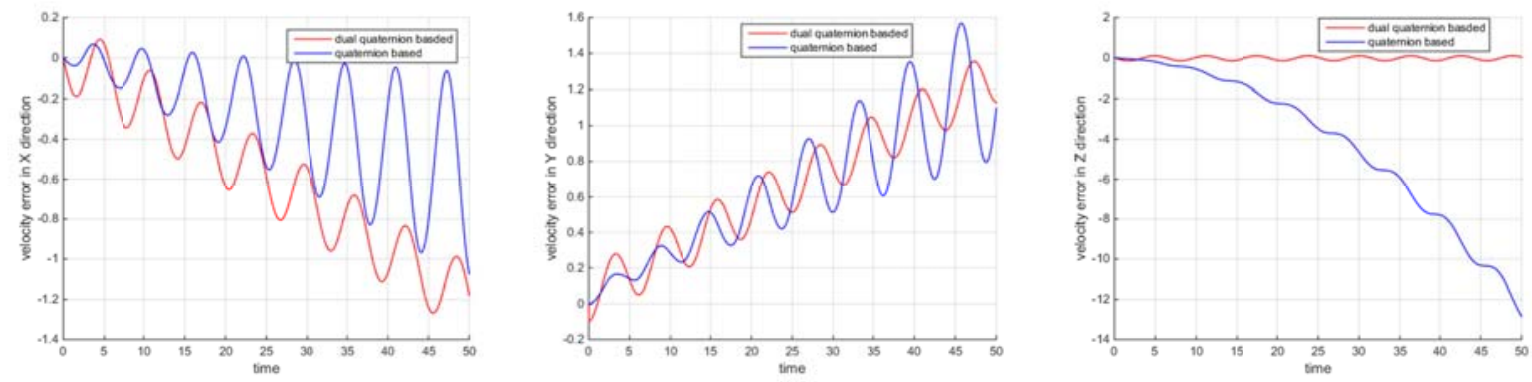

Fig. 2 Velocity error using IMU simulated data without adding noise

\subsection{Simulation With Noise}

The Gaussian noise added to original gyro and accelerometer simulated data can corrupt the precision of the integral of inertial differential equations quickly. Therefore, EKF framework is implemented to correct the estimated state.

The position is initialized at $\left[\begin{array}{lll}0 & 10 & 0\end{array}\right]^{T}$, velocity at $\left[\begin{array}{lll}10 & 0 & 10\end{array}\right]^{T}$ and attitude angle at $\left[\begin{array}{lll}0 & 0 & 0\end{array}\right]^{T}$. The gyro noise covariance is set with the value of $0.01 \mathrm{rad}^{2} / \mathrm{s}^{2}$, accelerometer noise covariance $0.25 \mathrm{~m}^{2} / \mathrm{s}^{4}$, the measurement position covariance $1 \mathrm{~m}^{2}$ and the measurement attitude angle covariance $9 \mathrm{deg}^{2}$.
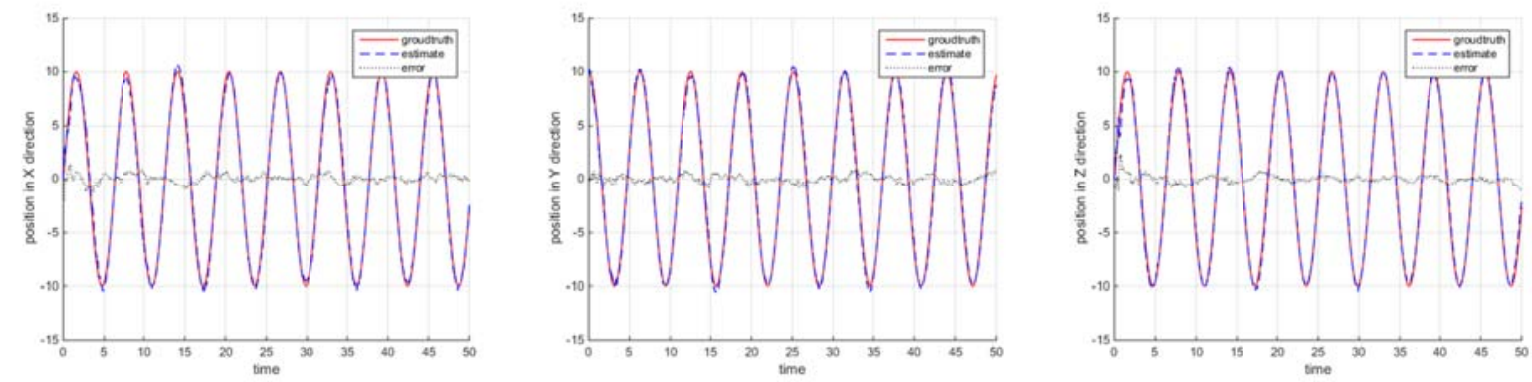

Fig. 3 Position estimation using fusion framework

Fig. 3 shows that the performance of proposed fusion framework in position estimate and the error converges to a steady state quickly. According to Fig. 4 Attitude estimation using fusion framework, the difference between real attitude angle and estimated value fall in the interval between -0.05 and 0.05 . Violent movements give rise to error peak. Hence, dual quaternion can be implemented in EKF framework. 

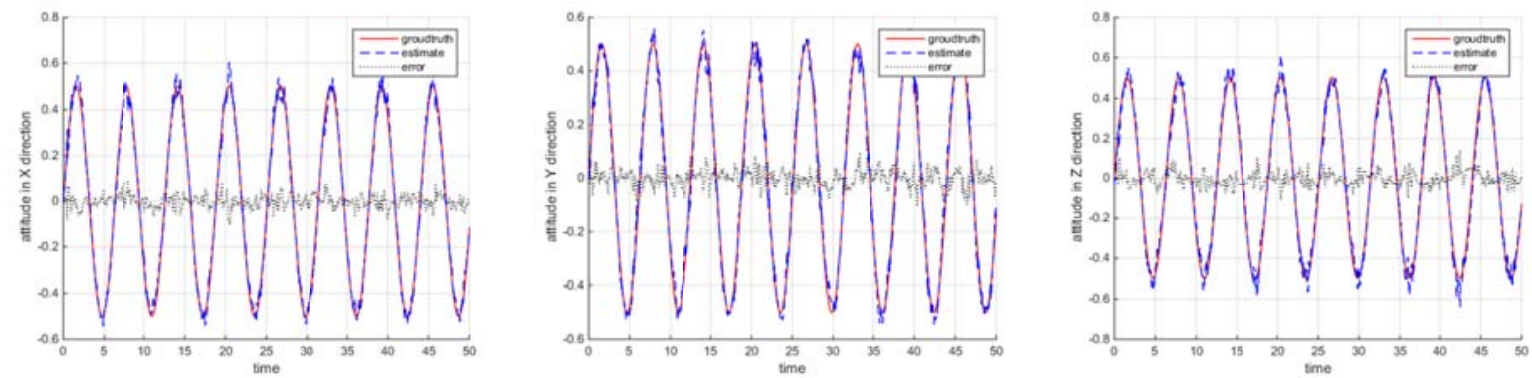

Fig. 4 Attitude estimation using fusion framework

\section{Conclusions}

A dual quaternion based fusion algorithm for estimating motion states of MAVs is proposed in this paper. The experiments have been performed with simulated data. Dual quaternion based inertial differential dynamics can capture coupling between translation and rotation more accurately compared to quaternion based method. It improves the precision of estimated results especially in the velocity and position estimation in altitude direction. In the fusion framework, the dual quaternion can be successfully implemented in EKF procedure. In future work, multiplicative error states based on dual quaternion will be explored to improve the performance and effectiveness of algorithm.

\section{References}

[1] Weiss S, Achtelik M W, Lynen S, et al. Monocular Vision for Long-term Micro Aerial Vehicle State Estimation: A Compendium[J]. Journal of Field Robotics, 2013, 30(5):803-831.

[2] Weiss S, Achtelik M W, Chli M, et al. Versatile distributed pose estimation and sensor self-calibration for an autonomous MAV[C]// IEEE International Conference on Robotics \& Automation. IEEE, 2012:31-38.

[3] Chilian A, Hirschmuller H, Gorner M. Multisensor data fusion for robust pose estimation of a six-legged walking robot[C]// IEEE/RSJ International Conference on Intelligent Robots \& Systems. 2011:2497-2504.

[4] Filipe, N, Kontitsis, M, Tsiotras, P. Extended Kalman Filter for spacecraft pose estimation using dual quaternions[J]. Journal of Guidance Control and Dynamics, 2015, 38:1-17.

[5] B. Kenwright, "A beginners guide to dual-quaternions: what they are,how they work, and how to use them for 3d character hierarchies," 2012.

[6] J. Vince, Quaternions for Computer Graphics. Springer, 2011. 